\title{
Motor performance of the hand in patients with rheumatoid arthritis
}

\author{
Kari Kauranen, Pekka Vuotikka, Markku Hakala
}

Department of

Physical Medicine and

University Central

Hospital, Oulu,

Finland

K Kauranen

Division of

Rheumatology,

Department of

Oulu University

Central Hospital

P Vuotikka

M Hakala

Correspondence to: Dr Kari Kauranen,

Department of Physical

Medicine and Rehabilitation,

Oulu University Central

Hospital, Kajaanintie 50,

90220 Oulu, Finland

Email:

kari.kauranen@ppshp.fi

Accepted for publication 21 March 2000
Rehabilitation, Oulu

Internal Medicine,

\begin{abstract}
Objectives-To examine the motor performance of the hand in a sample of patients with rheumatoid arthritis (RA). Subjects-The patient group comprised 21 (two men, 19 women) patients with RA. Twenty one control subjects matched for age and sex were selected from a larger reference group, which had been drawn from the local population.

Methods-The measured motor performance aspects were simple reaction time, choice reaction time, speed of movement, finger tapping speed, and coordination (that is, speed of movement/accuracy). Results were compared for age and sex matched pairs. The measurements were made with the Human Performance Measurement/Basic Elements of Performance system, which is a multifunctional system designed to measure different motor aspects of the hands, including reaction time, movement speed, tapping speed, and coordination.

Results-A comparison of the results for the patient and control groups indicated that the motor functions of patients with RA were impaired in all the measured aspects (with the exception of the index finger tapping test). The difference between the groups varied between $11 \%$ and $21 \%$ for the reaction time tasks, between $12 \%$ and $18 \%$ for the speed of movement tasks, and between $15 \%$ and $17 \%$ for the coordination task.

Conclusion-Based on the results of our research, it seems that $\mathrm{RA}$ decreases some motor performance functions of the hand expressed as simple reaction time, choice reaction time, speed of movement, and
\end{abstract}

Table 1 Demographic and clinical data of the patients with rheumatoid arthritis

\begin{tabular}{|c|c|c|c|c|c|}
\hline Variable & Mean & $S D$ & Range & Median & $\begin{array}{l}95 \% \text { CI for } \\
\text { median }\end{array}$ \\
\hline Age (years) & 53.1 & 12.0 & $33-75$ & 54 & 44 to 62 \\
\hline Weight $(\mathrm{kg})$ & 60.3 & 8.5 & $48-75$ & 62 & 53 to 65 \\
\hline Height $(\mathrm{cm})$ & 161.6 & 5.4 & $152-172$ & 162 & 158 to 165 \\
\hline Disease duration (years) & 16.8 & 11.4 & $1-33$ & 18 & 5 to 27 \\
\hline No of DMARDs $\star$ to date & 4.6 & 2.1 & $0-10$ & 4 & 4 to 5 \\
\hline Inflamed joints (n) & 8.9 & 6 & $1-26$ & 8 & 5 to 14 \\
\hline Tender joints (n) & 9.3 & 7.0 & $1-28$ & 8 & 4 to 15 \\
\hline Tender joints (hand) (n) & 4.7 & 4.2 & $0-17$ & 4 & 1 to 7 \\
\hline Swollen joints (hand) (n) & 5.9 & 4.6 & $0-21$ & 5 & 3 to 8 \\
\hline $\mathrm{ESR}^{\star}$ & 39.9 & 24.3 & $14-110$ & 31 & 25 to 48 \\
\hline $\mathrm{CRP}^{\star}$ & 30.5 & 21.9 & $5-70$ & 23 & 13 to 54 \\
\hline Pain VAS ${ }^{\star}(\mathrm{mm})$ & 35 & 26 & $0-80$ & 40 & 10 to 53 \\
\hline Grip strength $(\mathrm{kPa})$ & 26.7 & 26.6 & $0-115$ & 18 & 10 to 40 \\
\hline $\mathrm{KFT}^{\star}$ hand (score) & 21.6 & 14.1 & $0-40$ & 26 & 8 to 35 \\
\hline KFT shoulder (score) & 3.7 & 3.6 & $0-10$ & 3 & 0 to 7 \\
\hline KFT total (arm) (score) & 25.3 & 16.7 & $0-48$ & 30 & 9 to 41 \\
\hline
\end{tabular}

${ }^{\star}$ DMARDs $=$ disease modifying antirheumatic drugs; $\mathrm{ESR}=$ erythrocyte sedimentation rate; $\mathrm{CRP}$ $=\mathrm{C}$ reactive protein; VAS $=$ visual analogue scale; KFT $=$ Keitel function test. coordination. The changes were emphasised in movements performed with several joints.

(Ann Rheum Dis 2000;59:812-816)

Rheumatoid arthritis (RA) causes reduced functional capacity, ${ }^{1}$ which leads to difficulties in activities of daily living. Inflammatory and destructive changes of the joints may cause pain and decrease the range of motion in joints, and inflict periods of immobilisation, resulting in muscular atrophy. ${ }^{2}$ Many previous investigators have studied the muscle strength of patients with RA, and the impaired muscle strength and functions of patients with RA compared with those of healthy controls have been known for several years. ${ }^{3-5}$ However, the authors of the previous studies have given little attention to the effects of RA on other components of the performance of the hands, such as coordination and motor performance.

In one of the rare motor performance studies dealing with patients with RA, Ginsburg et al studied cognitive functions (including switching attention and hand-eye coordination tasks) in patients with systemic lupus erythematosus (SLE) or RA and noticed that the patients with SLE had poorer performance in tests measuring attention and visuospatial ability than patients with RA. ${ }^{6}$ However, they did not have healthy controls for comparison.

As RA causes functional limitations in the joints, we proposed that this might affect the movements or the movement patterns of the damaged and inflamed joints and designed a study to investigate these possible movement changes in five different tasks.

The purpose of the study was to examine the motor performance of the hand in a sample of patients with RA. The measured motor performance aspects were simple reaction time, choice reaction time, speed of movement, finger tapping speed, and coordination (that is, speed of movement/accuracy).

Subjects and methods

SUBJECTS

Patient group

The patient group comprised 21 patients with RA (two men, 19 women) admitted to hospital ${ }^{7}$ for orthopaedic surgery (11 for the hands and six for the feet) or for conservative treatment of their RA (four patients). At the time of the study, 18/21 (86\%) patients used some disease modifying antirheumatic drug (DMARD), five of them received a combination of drugs. The patients had no neurological symptoms. Table 1 gives detailed demographic and clinical data of the patients with RA. All patients were 
classed as right handed as this was their preferred hand for writing.

\section{Control group}

The control group matched for age and sex was selected from a larger reference group, which had been drawn earlier (1992) from a local population. ${ }^{8}$ In the earlier reference group study, the aim was to recruit 200 healthy subjects. To achieve this target, 500 subjects (50 in each age decade and sex group) were randomly selected (an age- and sex-specified sample was ordered from the Finnish Census Bureau in February 1992) from among the population of Oulu (population on 1 January 1992 was 102280 inhabitants). Then 343 letters were sent in the order of the names on the list to recruit 200 healthy volunteers of the required age and sex (response rate 58\%). The main criterion for inclusion was normal motor function and an ability to perform the tests. The subjects had different socioeconomic and educational backgrounds, and preferred handedness was self reported by the subjects. The sample comprised 100 women and 100 men, categorised by sex and age decade into 10 groups (for example, 21-30 year old men). Each group comprised 20 subjects.

Twenty one control subjects matched for age (mean (SD) 53.1 (12.1) years, range 33-75) and sex (two men, 19 women) were selected from the reference study sample. A suitable control subject was found for each patient with $\mathrm{RA}$ when the age range \pm 1 year was used. When two or more aged matched controls were available (four cases), the control subject was drawn at random. The mean (SD) height of the control group was $162.3(9.3) \mathrm{cm}$, range 150-192, and the mean (SD) weight was $64.7(12.0) \mathrm{kg}$, range 53-105. All patients were classed as right handed as this was their preferred hand for writing.

\section{TEST PROCEDURE}

The study was based on a cross sectional design, in which the patients with RA and controls were measured once. The same person measured all subjects, and standardised in-

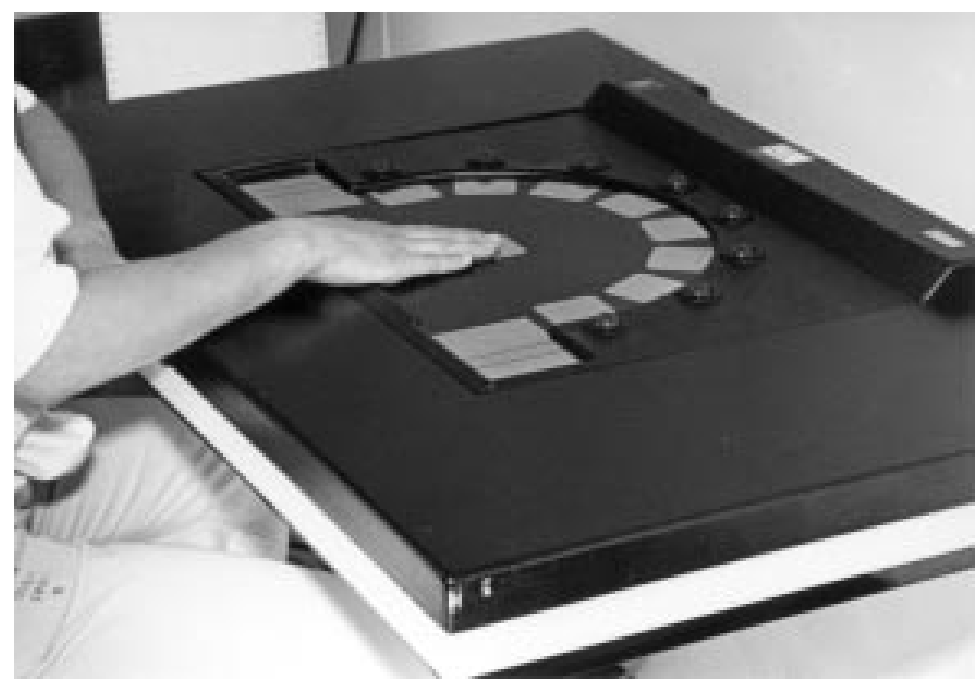

Figure 1 Human Performance Measurement/Basic Elements of Performance device and the measurement of reaction time of the hand. structions and explanations of the testing procedure were given to the subjects. Pairs matched for age and sex were set up, and the results for the patient and control groups were compared.

Clinical examination of the patients with RA included measurements of the number of tender and swollen joints, and pain (visual analogue scale (VAS)). ${ }^{9}$ In addition, grip strength of the dominant hand (Martin vigometer) was measured. The Keitel function test (KFT), ${ }^{10}$ a well known measure of impairment in RA, was used to assess various functional patterns of the arms and legs. The original KFT involves a series of 24 range of motion tasks performed by the patient, and it was used here with minor modifications, ${ }^{11}$ but only the arm tests were included for the present series. The total arm KFT (0-52) was divided into two subgroups of joints, one representing the hands and wrists $(0-42)$ and the other the shoulders $(0-10)$. The laboratory tests included measurements of erythrocyte sedimentation rate and $\mathrm{C}$ reactive protein.

The subjects signed an informed consent form agreeing to participate voluntarily in the study, and the study design was accepted by the Oulu University ethics committee.

The Human Performance Measurement/ Basic Elements of Performance (HPM/BEP) system (Human Performance Measurement, Arlington, TX 76004-1996) was used for the collection of motor performance data. In this study the module for the hands (BEP 1) was used. The module for hands (BEP 1) (fig 1) is a multifunctional system designed to measure different motor aspects of the hands, including reaction time, movement speed, tapping speed, and coordination. BEP 1 consists of eight red lights for visual stimuli and 15 touch-sensitive plates that are divided into four regions on top of the module. Different tests are performed on the four regions of the module, and all the tests are performed in a sitting position.

The same person measured all the subjects and controls, and standardised instructions and explanations of the testing procedures were given to the subjects. All procedures were as described in the manual. ${ }^{12}$ The tests were demonstrated, and each subject was allowed to perform some training trials of each test before the measured trials. The trials with anticipation errors or obvious delays were considered to have failed and were repeated. The subjects performed the tests in the same order, and the number of trials and the measurement times were set by the Human Performance Measurement software (HPM/BEP, version 4.2).

The test-retest reliability of HPM/BEP 1 tests has been described in detail by Kauranen and Vanharanta, ${ }^{8}$ and is acceptable. The participants in the reliability study (40 women) were healthy staff members aged 23-53, mean (SD) 33.8 (6.6), at the Oulu University Central Hospital. All the subjects were measured twice on consecutive days, and they performed the same tests on both days. All the subjects were tested for their right side. In the reliability study, the standard error of measurement 
(SEM) and the intraclass correlation coefficient of reliability (ICC) ${ }^{13}$ values were two choice reaction time: SEM 17.4, ICC 0.75, speed of movement: SEM 8.8, ICC 0.91, tapping speed: SEM 0.2, ICC 0.91, and coordination: SEM 0.5, ICC 0.81 .

The test subjects performed the following tests during one measurement session:

- Simple reaction time (five trials)

- One choice reaction time + speed of movement (five trials)

- Two choice reaction time + speed of movement (six trials)

- Index finger tapping speed (two trials)

- Coordination test (two trials).

Simple reaction time test

The test subject was instructed to place her/his hand on the plate, which was situated in the middle of the BEP 1 . The test subject then heard a beep signal, which was the sign to be ready for response. Two to six seconds after the acoustic sign all the eight lights were activated simultaneously and the test subject lifted her/his hand as quickly as possible from the plate with a dorsiflexion movement of the wrist joint. Reaction time was expressed in milliseconds, as the time from initiating a light stimulus to the time when the subject lifted her/his hand from the plate. The subjects performed five trials.

\section{One choice reaction time and speed of movement} test

The subject heard a beep signal, which was the indication for her/him to be ready for response. Two to six seconds after the acoustic signal a light stimulus appeared, and the subject was instructed to lift her/his hand immediately after the predefined light stimulus appeared and to move it as quickly as possible to the plate immediately in front of the activated light. Two different measures were obtained from each trial: (a) one choice reaction time, expressed in milliseconds, is the time between the appearance of a light stimulus and the hand lifting from the centre plate; (b) movement speed, expressed in $\mathrm{cm} / \mathrm{s}$, is determined as the distance between the centre plate and the target plate (distance $10 \mathrm{~cm}$ ) divided by the time needed for the performance of the movement. The subjects performed five trials.

\section{Two choice reaction time and speed of movement test}

The subject performed the test with the same method as in the one choice reaction time and the speed of movement test, but there were two possible activated lights (measurement system presented stimuli in a pseudo-random order). The subjects performed six trials.

Index finger tapping test

The subject tapped the touch plate with the maximum rate of her/his index finger for 10 seconds. The results were expressed as taps/s. The subjects performed two trials.

Coordination test

The coordination test was a modified Fitts' task, ${ }^{14}$ where the movement amplitude and tar- get width were constants. The subject was instructed to tap two narrow plates alternately (the width of the plates was $17 \mathrm{~mm}$ and the distance between them $40 \mathrm{~cm}$ ) as rapidly as possible and to avoid errors for a period of 10 seconds. Two different measures were obtained from each trial: (a) a measure of accuracy expressed as the percentage of correct hits; and (b) a measure of the average movement speed during the task given in units of $\mathrm{cm} / \mathrm{s}$. The final measure was obtained by combining speed and accuracy, and the result was expressed as bits/s. ${ }^{14}$ The subjects performed two trials.

STATISTICAL METHODS

Mean value, SD, and $95 \%$ confidence intervals of the difference of each test were calculated and presented. A non-parametric paired $t$ test (Wilcoxon test for matched pairs) was used to determine whether there was a statistically significant difference between the values of the matched pairs. We used non-parametric statistics rather than parametric statistics because the group consisted of 21 subjects. Parametric statistics require a normal distribution and with "only" 21 subjects this requirement was not fulfilled every time. The correlation between the motor performance tests and the VAS score, the KFT score, grip strength, disease duration, and the number of tender and swollen joints was tested with Spearman's correlation coefficient.

For all statistical tests, the 0.05 level of probability was accepted as the criterion for significance. The statistical software used were the SOLO (version 4.0) (BMDP Statistical Software Inc) and the Statistical Package for Social Sciences (SPSS, version 7.0).

\section{Results}

No statistically significant differences in the results by age, sex, weight, and height were found between the patients with RA and the controls, and hence the groups were comparable and equal in these respects.

The results indicated that the reaction times were longer in the group of patients with RA than in the control group on both sides. In the speed of movement tests the patients with RA were slower than the controls on both sides. There were no statistically significant differences in the index finger tapping speed between the groups, but the coordination values of the hands were lower in the patients with RA on both sides. Table 2 presents detailed results of the motor performance tests.

There was no correlation between the motor performance tests and the VAS score, the KFT score, grip strength, disease duration, and the number of swollen joints. However, the speed of movement (lateral direction) correlated with the number of tender joints of the hands (Spearman's correlation coefficient $\left(r_{\mathrm{s}}\right)=$ $-0.44, \mathrm{p}=0.048$ for the right hand, and $r_{\mathrm{s}}=-0.50, \mathrm{p}=0.021$ for the left hand). In addition, speed of movement of the right hand tended to correlate with the total KFT score of the arm $\left(r_{\mathrm{s}}=-0.41, \mathrm{p}=0.06\right.$ in the forward direction test and $r_{\mathrm{s}}=-0.36, \mathrm{p}=0.09$ in the lateral direction test). 
Table 2 Motor performance results in the patients with rheumatoid arthritis $(R A)$ and control groups $(n=21)$

\begin{tabular}{|c|c|c|c|c|c|c|c|}
\hline Measurement & Side & Unit & $\begin{array}{l}\text { Patients with } R A \\
\text { (mean }(S D))\end{array}$ & $\begin{array}{l}\text { Controls } \\
(\text { mean }(S D))\end{array}$ & $\begin{array}{l}95 \% \text { CI for the } \\
\text { difference of mean }\end{array}$ & $p$ Value & $\begin{array}{l}\text { Difference between } \\
\text { groups (\%) }\end{array}$ \\
\hline Simple reaction time & Right & $(\mathrm{ms})$ & $217(44)$ & $192(30)$ & 4 to 45 & 0.0173 & +13 \\
\hline Simple reaction time & Left & $(\mathrm{ms})$ & $208(30)$ & $187(27)$ & 4 to 39 & 0.0129 & +11 \\
\hline One choice reaction time & Right & $(\mathrm{ms})$ & $272(46)$ & $245(46)$ & 4 to 50 & 0.0199 & +11 \\
\hline One choice reaction time & Left & (ms) & $272(57)$ & $235(36)$ & 13 to 62 & 0.0028 & +16 \\
\hline Two choice reaction time & Right & $(\mathrm{ms})$ & $350(77)$ & $312(43)$ & 8 to 69 & 0.0276 & +12 \\
\hline Two choice reaction time & Left & $(\mathrm{ms})$ & $354(77)$ & $293(46)$ & 23 to 101 & 0.0037 & +21 \\
\hline Speed of movement (forward) & Right & $(\mathrm{cm} / \mathrm{s})$ & $83(24)$ & $96(27)$ & -26 to 0 & 0.0458 & -14 \\
\hline Speed of movement (forward) & Left & $(\mathrm{cm} / \mathrm{s})$ & $78(21)$ & $95(31)$ & -31 to 0 & 0.0355 & -18 \\
\hline Speed of movement (lateral) & Right & $(\mathrm{cm} / \mathrm{s})$ & $88(27)$ & $103(27)$ & -33 to 1 & 0.0150 & -15 \\
\hline Speed of movement (lateral) & Left & $(\mathrm{cm} / \mathrm{s})$ & $82(31)$ & $93(26)$ & -27 to 5 & 0.0263 & -12 \\
\hline Tapping speed & Right & (taps/s) & $5.01(0.82)$ & $5.37(0.98)$ & -0.95 to 0.19 & 0.1842 & -7 \\
\hline Tapping speed & Left & (taps/s) & $4.91(0.77)$ & $4.90(0.97)$ & -0.58 to 0.60 & 0.8563 & 0 \\
\hline Coordination & Right & (bits/s) & $8.22(1.49)$ & $9.65(1.29)$ & -2.17 to -0.69 & 0.0024 & -15 \\
\hline Coordination & Left & (bits/s) & $7.58(1.52)$ & $9.16(0.98)$ & -2.40 to -0.78 & 0.0017 & -17 \\
\hline
\end{tabular}

\section{Discussion}

The purpose of the study was to examine the motor performance of the hand in a sample of patients with RA. The measured motor performance aspects were simple reaction time, choice reaction time, speed of movement, finger tapping speed, and coordination. Comparison of the results for the patients with RA and for the healthy controls matched for age and sex, indicated that the motor functions of patients with RA were impaired in all the measured aspects (except the index finger tapping test). The difference between the groups varied between $11 \%$ and $21 \%$ in the reaction time tasks, between $12 \%$ and $18 \%$ in the speed of movement tasks, and between $15 \%$ and $17 \%$ in the coordination task.

The reason for these differences may be explained by the neuromuscular problems of the patients with RA. Previous studies have shown an association between RA and nerve function impairment, ${ }^{15}$ and it seems that RA accelerates muscle fibre degeneration, especially in fast-twitch muscle fibres. ${ }^{16}$ There is also a clear difference in the shape of the forcetime curve between patients with RA and healthy subjects at all force levels. Healthy subjects produce higher force levels in rapid isometric muscle action. ${ }^{17}$ In addition, one reason for the poorer performance of patients with RA may be that the destructive and inflammatory changes in joints and pain or fear of pain prevent the subjects from performing fast movements as quickly as normal.

All the measured motor performance aspects are essential and important for various daily activities, which constitute a substantial part of human life, and their importance is especially great in traffic, sports, and unexpected situations, but we found no previous motor performance studies on patients with RA with which to compare our results.

The results indicated that the reaction times were longer in the RA group than in the control group for both hands. Reaction time is a common method to study a person's central information processing speed and fast coordinated peripheral movement response. Reaction time tasks load and involve both the central and the peripheral components and functions. Our patients were severely impaired (for example, KFT was rather high), and it may be that the abovementioned lower motor unit problems and the destructive and inflammatory changes (for example, joint stiffness, deformations, and limitations in range of motion) in joints prevent patients with RA from performing fast movements as quickly as normal. In addition, the pain or fear of pain may delay these movements. We performed three reaction time tasks, and it should be noted that the difference between the groups did not increase when the number of possible choices increased (for example, in a two choice reaction time task), but the patients with RA only were slower.

The patients with RA were slower than the controls in the speed of movement tests. This finding might be mainly a consequence of unpleasant feelings of pain or fear of pain during movements (the speed of movement correlated with the number of tender joints of the hand), which leads to avoidance of fast movements. The speed of movement indicates the subject's ability to perform fast movements, which are important in different balance and protection movements and reflexes. Based on the results, this might even mean that patients with RA have an increased risk of falling because of their decreased ability to perform fast movements. This shortcoming together with the longer reaction times might be hazardous for rheumatoid patients, because osteoporosis is common among them, ${ }^{18}$ which would increase the relative risk of fractures in these patients after a fall. ${ }^{19}$

The coordination values of both hands were lower in the RA patient group. The coordination test (modified Fitts' task) involves a type of visually guided motor response, which is an essential component of a wide variety of skills needed for daily work and recreation. ${ }^{20}$ In coordination tests the final measure was obtained by combining speed and accuracy. The measure of accuracy was expressed as the percentage of correct hits, and the measure of average movement speed during the task given in units of $\mathrm{cm} / \mathrm{s}$. We analysed our coordination results in more detail and noticed that the mean values of speed of movement, in particular, were lower in the patients with RA. Thus it seems that the decrease in total coordination is caused mainly by a decrease in the speed of movement component. This may be explained by physical impairment caused by RA and pain or fear of pain, as discussed earlier for reaction times and speed of movement. 
Results of the finger tapping test were an exception as there were no statistically significant differences between the groups in this task. This was surprising, because the movement is performed with one joint and the performance time is longer $(10 \mathrm{~s})$ than in the other motor performance tests, which were performed with a single fast movement. We expected that the differences between the groups would have became obvious, especially in a test of longer duration and with repetition of joint movements (for example, the tapping test). However, no such effect was seen. From these results it seems that the differences between the groups were emphasised in movements and tasks that were performed with several joints, and it might be that the condition of the larger joints, such as the shoulder and the elbow joints, have an important role in the fast movements and the total performance of the hand in patients with RA. In exercise therapy special attention should be paid to the movements of these joints.

There were no correlations between the motor performance tests and the VAS score, the KFT score, grip strength, disease duration, and the number of swollen joints. This finding was partly expected because the age range of our group of rheumatoid patients (33-75 years) was so wide, and age explains a considerable portion of the motor performance values $^{8}$ and upsets other relations (the effect of age was controlled with partial correlation analysis). However, it should be noted that the speed of movement (lateral direction) correlated with the number of tender joints of the hands, and the speed of movement of the right hand tended to correlate with the total KFT score of the arm.

STUDY LIMITATIONS

Although we confirmed the hypothesis based on the abovementioned results and rejected the null hypothesis, the number of subjects in this study was quite small and the results might have been more conclusive if a larger sample of subjects had been used. Secondly, the selection of subjects in this study was not random and they included mainly women, which limits the generalisation of the results.

It is also to be noted that our series represented a hospital based population with advanced RA. Consequently, their median level of arm KFT was rather high, indicating clearly impaired function. Overall, our data are preliminary and should be tested in a large RA series including early cases.

The control subjects were from various socioeconomic and educational categories. This helps somewhat to generalise the results, and the composition of the sample can be presumed to represent the normal population of the area. However, the subjects were invited by post for the measurements, and this might have caused some selection. The response rate $(58 \%)$ was quite low and this impairs somewhat the generalisation of the results. The precise reason for the low response rate is not known, but studying or working in other cities, shift work, and lack of time in the younger age groups might be possible explanations.

\section{Conclusion}

Based on the results of our research, it seems that RA decreases some motor performance functions of the hand expressed as simple reaction time, choice reaction time, speed of movement, and coordination. The changes were emphasised in movements performed with several joints.

1 Beals CA, Lampman RM, Banwell BF, Braunstein EM, Albers JW, Castor CW. Measurement of exercise tolerance in patients with rheumatoid arthritis and osteoarthritis. J Rheumatol 1985;12:458-61.

2 Herbison GJ, Ditunno JF, Jaweed MM. Muscle atrophy in rheumatoid arthritis. J Rheumatol 1987;14(suppl 15):7881

3 Tiselius P. Studies on joint temperature, joint stiffness and muscle weakness in rheumatoid arthritis. Acta Rheumatologica Scandinavica 1969;suppl 14:1-106

4 Ekblom B, Lövgren O, Alderin M, Fridström M, Satterström G. Physical performance in patients with rheumatoid arthritis. Scand J Rheumatol 1974;3:121-5.

5 Häkkinen A, Hannonen P, Häkkinen K. Muscle strength in healthy people and in patients suffering from recent-onset inflammatory arthritis. Br J Rheumatol 1995;34:355-60.

6 Ginsburg KS, Wright EA, Larson MG, Fossel AH, Albert $\mathrm{M}$, Schur PH, et al. A controlled study of the prevalence of cognitive dysfunction in randomly selected patients with systemic lupus erythematosus. Arthritis Rheum 1992;35: 776-82.

7 Arnett FC, Edworthy SM, Bloch DA, McShane DJ, Fries $\mathrm{JF}$, Cooper NS, et al. The American Rheumatism Association 1987 revised criteria for the classification of rheumatoid arthritis. Arthritis Rheum 1988;31:315-24.

8 Kauranen K, Vanharanta H. The influence of aging, gender and handedness on the motor performance of upper and and handedness on the motor performance of upper
lower extremities. Percept Mot Skills 1996;82:515-25.

9 Scott J, Huskinsson E. Graphic representation of pain. Pain 1976;2:175-84.

10 Keitel W, Hoffmann H, Weber G, Krieger U. Ermittlung der prozentualen Funktionsminderung der Gelenke durch einen Bewegungsfunktionstest in der Rheumatologie. Deutsche Gesundsheitswesen 1971;26:1901-3.

11 Hakala M, Nieminen P. Functional status assessment of physical impairment in a community based population with rheumatoid arthritis: severely incapacitated patients are rare. J Rheumatol 1996;23:617-23.

12 Kondraske G. HPM/BEP manual. 3rd printing. Arlington: Human Performance Measurement, 1991.

13 Fleiss JL. The design and analysis of clinical experiments. New York: Wiley 1986:2-12.

14 Fitts PM. The information capacity of the human motor system in controlling the amplitude of movement. J Exp Psychol 1954;47:381-91.

15 Haslock D, Wright V, Harriman D. Neuromuscular disorders in rheumatoid arthritis. Q J Med 1970;39:33558.

16 Danneskiold-Samsoe B, Grimby G. Isokinetic and isometric muscle strength in patients with rheumatoid arthritis. The relationship to clinical parameters and the influence of corticosteroid. Clin Rheumatol 1986;5:459-67.

17 Häkkinen A, Sokka T, Kotaniemi A, Paananen M-L, Mälkiä $\mathrm{E}$, Kautiainen $\mathrm{H}$, et al. Muscle strength characteristics and central bone mineral density in women with recent onset rheumatoid arthritis compared with healthy controls. Scand J Rheumatol 1999;28:1-7.

18 Hansen M, Florescu A, Stoltenberg M, Podenphant J, Pedersen-Zbinden B, Horslev-Petersen K, et al. Bone loss in rheumatoid arthritis. Influence of disease activity, duration of the disease, functional capacity, and corticosteroid treatment. Scand J Rheumatol 1996;25:367-76.

19 Hooyman J, Melton L, Nelson A, O'Fallon W, Riggs B. Fractures after rheumatoid arthritis. Arthritis Rheum Fractures after rhe

20 Wickens CS. Engineering psychology and human performance. Columbus: Merrill, 1984. 